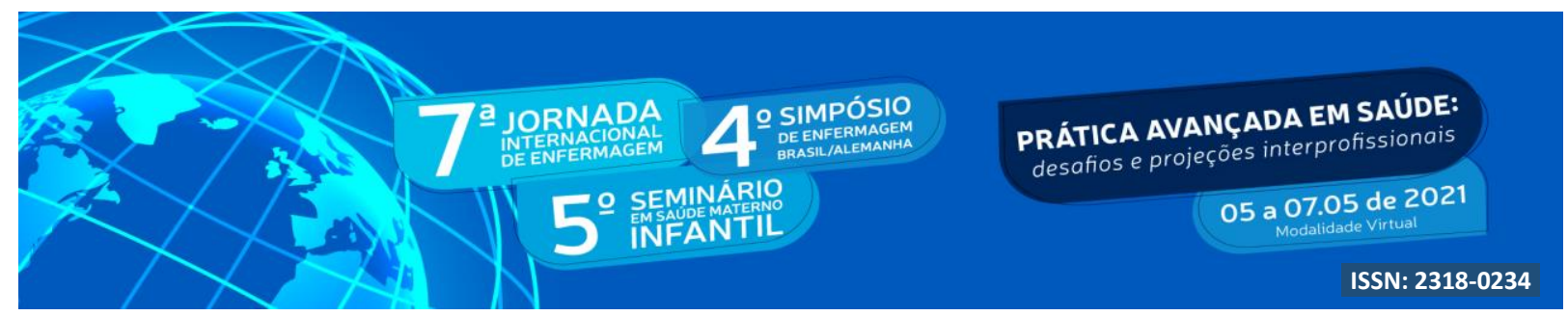

DOI: http://doi.org/10.48195/jie2021-114

\title{
APLICATIVOS MÓVEIS DIRECIONADOS ÀS GESTANTES: REVISÃO INTEGRATIVA
}

\author{
Andressa Ferreira Portoㅁ; Fátima Helena do Espírito Santo²; Carlos Frank viga \\ Ramos$^{3}$; Cleisiane Xavier Diniz ${ }^{4}$; Maria de Nazaré de Souza Ribeiro ${ }^{5}$
}

\begin{abstract}
RESUMO
Objetivo: Identificar as contribuições das tecnologias educacionais com uso de aplicativos móveis para gestantes. Metodologia: Trata-se de um estudo de revisão integrativa da literatura realizada na base de dados: LILACS, MEDLINE/PUBMED, SCIELO e nas bibliotecas BVS. Para estratégia de busca foram utilizados descritores em saúde. Após o cruzamento dos descritores e aplicação de critérios de inclusão, exclusão e de elegibilidade, resultaram em 14 estudos. Resultados: Evidenciou-se 14 artigos, que evidenciaram melhorias no cuidado e na vida das mulheres que faziam o uso dos aplicativos, e essas tecnologias mostraram de forma educativa, informações e diversos conteúdos sobre a gestação. Conclusão: Presente estudo mostrou que a tecnologia educativa móvel é tanto um importante instrumento para os profissionais de saúde, quanto para as usuárias gestantes. Ainda existe indicadores que lacunas precisam ser preenchidas quanto a qualidade de informações, eficácia das orientações, informações audiovisuais instrutivas e a usabilidade dos aplicativos móveis.
\end{abstract}

Palavras-chave: Enfermagem; Gravidez; Tecnologia móvel.

\begin{abstract}
Objective: To identify the contributions of educational technologies with the use of mobile applications for pregnant women. Methodology: This is an integrative literature review study carried out in the database: LILACS, MEDLINE / PUBMED, SCIELO and in the VHL libraries. For search strategy, health descriptors were used. After crossing the descriptors and applying inclusion, exclusion and eligibility criteria, they resulted in 14 studies. Results: There were 14 articles, which showed improvements in the care and life of women who used the applications, and these technologies showed, in an educational way, information and various contents about pregnancy. Conclusion: This study showed that mobile educational technology is both an important tool for health professionals and pregnant users. There are still indicators that gaps need to be filled in terms of the quality of information, the effectiveness of guidelines, instructive audiovisual information and the usability of mobile applications.Key Words: Mobile technology; Nursing; Pregnancy.
\end{abstract}

\footnotetext{
${ }^{1}$ Estudante do Curso de Mestrado Profissional em Enfermagem Assistencial. Universidade Federal Fluminense. E-mail: andressafp@id.uff.br

${ }^{2}$ Orientadora. Doutora. Universidade Federal Fluminense. E-mail: fatahelens@ gmail.com

${ }^{3}$ Coorientador. Doutor. Universidade Federal do Acre. E-mail: carlos.ramos@ufac.br

${ }^{4}$ Estudante do curso de pós-doutorado. Universidade Federal Fluminense. E-mail: cxdiniz@ gmail.com

${ }^{5}$ Docente. Doutora. Universidade do Estado do Amazonas. E-mail: mnribeiro2@gmail.com
} 


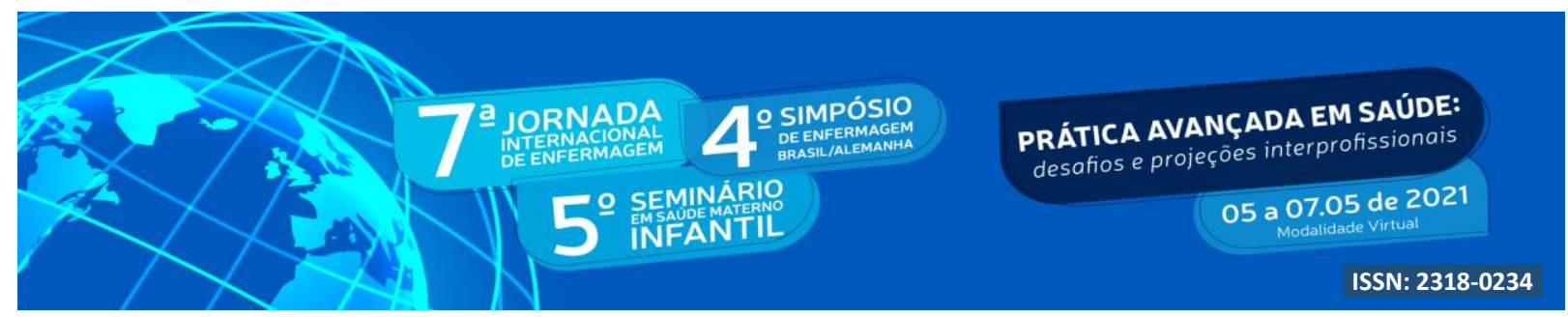

\section{INTRODUÇÃO}

Os seres humanos têm presenciado diferentes modos de comunicar-se, com o avanço da tecnologia, o mundo adaptou-se à nova forma de interagir com diversos públicos, seja no âmbito educacional, empresarial, entretenimento, saúde e outras variedades de implantação (MENDONÇA et al. 2017).

Segundo Kennelly et al. (2018) as tecnologias apresentam desenvolvimento admirável para o campo da Saúde. O aumento do consumo de telefones celulares e smartphones, trazem melhorias das condições de saúde dos usuários, independente da faixa etária, alcançando dessa forma vários grupos populacionais.

A educação em saúde consiste em um instrumento de implantação de estratégias e ações de promoção à saúde, que busca melhorar a qualidade de vida das pessoas, é visto como um método de interesse para a população. A criação de aplicativos (App's) para dispositivos móveis, tornou-se um fator favorável dessas práticas. Passou a fazer parte da rotina da população, a partir da facilidade de acesso à internet através da conexão sem fio (wi-fi). (EDWARDS et al. 2016).

Segundo Candau (1978) as tecnologias Educacionais são estratégias para inovar a educação. No âmbito da saúde, a tecnologia pode ser uma grande aliada a educação em saúde, pois consiste em ser um instrumento de grande importância, que facilita a orientação e comunicação com os usuários. Desta forma, de grande valia e colaboração para a prática de educação em saúde.

Pode-se sugerir que a cerca da assistência de enfermagem, as tecnologias educacionais são ferramentas importantes a serem utilizadas no processo de ensino, como um meio de prover contribuições as pessoas que buscam conhecimento.

Diante dessas considerações, surgiu a seguinte questão de pesquisa: quais as contribuições das tecnologias educacionais com uso de aplicativos moveis para gestantes?

\section{OBJETIVO}

Identificar as contribuições das tecnologias educacionais com uso de aplicativos móveis para gestantes. 


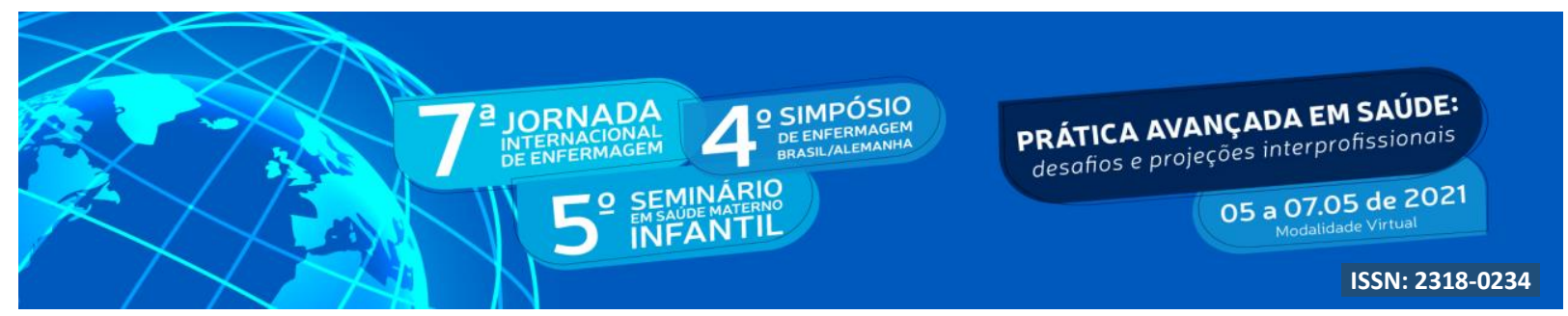

\section{METODOLOGIA}

Trata-se de um estudo de uma Revisão Integrativa da Literatura (RIL) que corresponde ao levantamento de evidências sobre determinado tema, sintetizando-o para elucidar dúvidas, contribuir para tomada de decisão, expressar lacunas e qualificar a prática profissional por meio da translação do conhecimento (MENDES et al. 2018).

Para composição da presente revisão foram percorridas as seguintes etapas: elaboração da questão norteadora; perscruta nas bases de dados ad otando critérios de inclusão e exclusão; extração dos dados relevantes; avaliação dos estudos; interpretação dos resultados e apresentação da revisão (MENDES et al. 2018).

Foi eleita a questão norteadora: Quais as contribuições das tecnologias educacionais com uso de aplicativos moveis para gestantes?

Os dados foram coletados no período de outubro e novembro de 2020, aos pares, em consulta ao Portal da Biblioteca Virtual em Saúde e ao Portal de Periódicos Capes, nas bases de dados Literatura Latino-Americana e do Caribe em Ciências da Saúde (Lilac's), Medical Literature Analysis and Retrieval System Online (Medline/PUBMED) e nas bibliotecas Portal da Biblioteca Virtual de Saúde (BVS) e Scientific Eletronic Library Online (Scielo).

Para viabilizar a busca, foi realizado consultas dos Descritores em Ciências da Saúde (DeSC) e noMedical Subject Headings (MeSH). Foram identificados os seguintes descritores em português e seus correspondentes em inglês: "Gravidez”, “tecnologia móvel”, “aplicativos móveis", "cuidados de enfermagem" e "enfermagem". Os descritores foram combinados entre si por meio do operador booleano AND e OR.

Os critérios de inclusão dos estudos foram: artigos originais que abordassem a temática do estudo, disponíveis online na íntegra e publicados nos últimos 10 anos em língua portuguesa, inglesa e espanhola. Os critérios de exclusão considerados foram: Resumos, estudos que não respondessem à pergunta norteadora, duplicidade de artigos, editoriais, teses, dissertações, trabalhos de conclusão de curso, estudos de revisão e relato de experiência.

Ao utilizar a estratégia de busca proposta foram encontrados 371 artigos, aos quais foram aplicados os critérios de inclusão, e excluídos os repetidos, restando 238, cujos títulos e resumos foram lidos. Após, restaram 31 para leitura completa, possibilitando a seleção de 14 


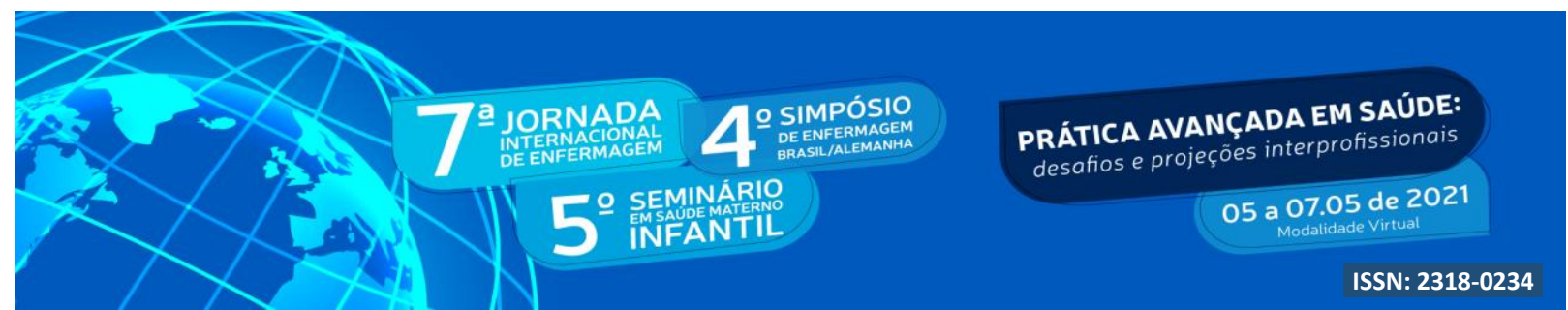

artigos que apresentaram adesão ao tema de pesquisa. No quadro 1 apresenta a seleção dos artigos segundo base de dados, tipo de tecnologia e critérios de elegibilidade.

Quadro 1 - Seleção dos artigos segundo base de dados, tipo de tecnologia e critérios de elegibilidade.

\begin{tabular}{|c|c|c|c|c|c|c|c|c|}
\hline $\begin{array}{c}\text { Base de } \\
\text { dados }\end{array}$ & $\begin{array}{c}\text { Seleção } \\
\text { pela } \\
\text { Estratégia } \\
\text { de busca }\end{array}$ & $\begin{array}{c}\text { Seleção } \\
\text { após } \\
\text { critérios de } \\
\text { elegibilidade }\end{array}$ & $\begin{array}{c}\text { Seleção } \\
\text { pós leitura } \\
\text { (título e } \\
\text { resumo) }\end{array}$ & $\begin{array}{c}\text { Exclusão } \\
\text { de } \\
\text { duplicados }\end{array}$ & $\begin{array}{c}\text { Exclusão } \\
\text { por } \\
\text { duplicidade } \\
\text { quanto ao } \\
\text { tipo de } \\
\text { tecnologia }\end{array}$ & $\begin{array}{l}\text { Exclusão } \\
\text { após } \\
\text { leitura na } \\
\text { íntegra }\end{array}$ & $\begin{array}{c}\text { Seleção } \\
\text { final }\end{array}$ \\
\hline & SCIELO & 107 & -- & -- & -- & -- & -- & -- \\
\hline & LILACS & 14 & 14 & 6 & 4 & 00 & 1 & 1 \\
\hline & MEDLINE & 250 & 224 & 25 & 1 & 00 & 11 & 13 \\
\hline
\end{tabular}

Fonte: Elaborado pelos autores. Brasil, 2020.

00 Nenhuma exclusão -- Nenhum artigo

\section{RESULTADOS E DISCUSSÃO}

A amostragem final contou com 14 artigos (Quadro 1) oriundos de periódicos internacionais $(85,71 \%)$ e nacionais $(14,29)$. Com relação ao ano de publicação teve por destaque o ano de 2019 (35,71\%) das publicações e os anos de 2018 e 2017 (28,57\%).

Em relação a base de dados, doze $(85,71 \%)$ estudos foram encontrados na Medline/PUBMED e apenas dois (14,28\%) foram encontrados na LILAC's. No quadro 2 apresenta a sumarização dos estudos selecionados apresentando título, autores, país, ano de publicação, objetivo e desfecho.

Quadro 2 - Sumarização dos estudos selecionados apresentando título, autores, país, ano de publicação, objetivo e desfecho.

\begin{tabular}{|c|c|c|c|c|}
\hline & Título & $\begin{array}{l}\text { Autores } \\
\text { País } \\
\text { Ano }\end{array}$ & Objetivo & Desfecho \\
\hline A1 & $\begin{array}{l}\text { Aplicativo móvel como } \\
\text { ferramenta de educação e } \\
\text { engajamento pré-natal }\end{array}$ & $\begin{array}{l}\text { Ledford CJW. et } \\
\text { al. } \\
\text { Holanda } \\
2016\end{array}$ & $\begin{array}{l}\text { Comparar a eficácia } \\
\text { de um aplicativo } \\
\text { móvel versus um } \\
\text { guia de caderno } \\
\text { espiral durante o } \\
\text { cuidado pré-natal }\end{array}$ & $\begin{array}{l}\text { Mostra que o uso do } \\
\text { aplicativo móvel é mais } \\
\text { eficaz do que cadernos, } \\
\text { além de ser uma } \\
\text { ferramenta com melhor } \\
\text { aceitacão das pacientes. }\end{array}$ \\
\hline
\end{tabular}




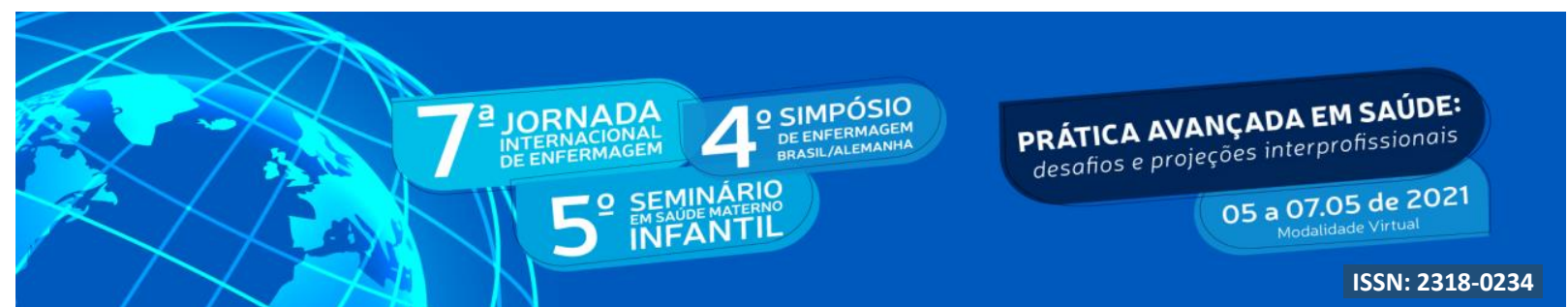

\begin{tabular}{|c|c|c|c|c|}
\hline A2 2 & $\begin{array}{l}\text { Aplicativo de smartphone para } \\
\text { mulheres } \\
\text { com diabetes mellitus } \\
\text { gestacional }\end{array}$ & $\begin{array}{l}\text { Borgen I. et al. } \\
\text { Inglaterra } \\
2017\end{array}$ & $\begin{array}{l}\text { Avaliar se o uso } \\
\text { do } \quad \text { aplicativo } \\
\text { Gravidez }+ \text {, além dos } \\
\text { cuidados padrão } \\
\text { resulta em melhores } \\
\text { níveis de glicose } 3 \\
\text { meses após o parto }\end{array}$ & $\begin{array}{l}\text { Intervenções estratégicas } \\
\text { para reduzir } \\
\text { hiperglicemia } \\
\text { para mulheres com DMG } \\
\text { pode prevenir Diabetes } \\
\text { Mellitos tipo } \\
\text { entre mulheres com DMG } \\
\text { ou DMG recente. O uso de } \\
\text { aplicativos } \\
\text { intervenções de saúde } \\
\text { parece apropriado. }\end{array}$ \\
\hline $\mathbf{A} \mathbf{3}$ & $\begin{array}{l}\text { Avaliação de um aplicativo de } \\
\text { nutrição e atividade física em } \\
\text { smartphone para fornecer } \\
\text { conselhos sobre estilo de vida a } \\
\text { mulheres grávidas }\end{array}$ & $\begin{array}{l}\text { Dodd JM. et al. } \\
\text { EUA } \\
2017\end{array}$ & $\begin{array}{l}\text { Avaliar o impacto de } \\
\text { um aplicativo de } \\
\text { smartphone como um } \\
\text { adjunto às consultas } \\
\text { para facilitar a } \\
\text { mudança na dieta e } \\
\text { na atividade física } \\
\text { entre as mulheres } \\
\text { grávidas. }\end{array}$ & $\begin{array}{l}\text { A adição de um aplicativo } \\
\text { de smartphone como um } \\
\text { complemento para um } \\
\text { estilo de vida das mulheres } \\
\text { grávidas, não foi associado } \\
\text { a melhorias significativas } \\
\text { na ingestão alimentar ou } \\
\text { atividade física. }\end{array}$ \\
\hline A4 & $\begin{array}{l}\text { Desenvolvimento e Avaliação } \\
\text { de um aplicativo de iPad para } \\
\text { promover o conhecimento de } \\
\text { uso e cessação de tabaco por } \\
\text { mulheres grávidas }\end{array}$ & $\begin{array}{l}\text { Dotson JAW. et } \\
\text { al. } \\
\text { Holanda } \\
2017\end{array}$ & $\begin{array}{lr}\text { Descrever } & \mathrm{o} \\
\text { desenvolvimento } & \mathrm{e} \\
\text { avaliação de um } & \text { um } \\
\text { aplicativo para } & \text { iPad } \\
\text { para promover } & \text { o } \\
\text { conhecimento sobre } \\
\text { o risco do tabagismo } \\
\text { e recursos para } \\
\text { cessação } & \text { para } \\
\text { grávidas. }\end{array}$ & $\begin{array}{l}94 \% \text { concordaram que } \\
\text { ajudou a compreender os } \\
\text { riscos do tabaco; } \\
75 \% \text { relatou que as } \\
\text { informações ajudaram a } \\
\text { querer } \\
\text { pararam de fumar; Um } \\
\text { iPad e uma aplicação pode } \\
\text { ser uma ferramenta útil na } \\
\text { educação sobre cessação } \\
\text { do tabagismo durante a } \\
\text { gravidez. }\end{array}$ \\
\hline A5 & $\begin{array}{l}\text { Eficácia da tecnologia móvel e } \\
\text { ganho de peso em mulheres } \\
\text { grávidas em Callao, Peru }\end{array}$ & $\begin{array}{c}\text { Mamani TC. et } \\
\text { al. } \\
\text { Colômbia } \\
2017\end{array}$ & $\begin{array}{l}\text { Avaliar a eficácia da } \\
\text { tecnologia móvel no } \\
\text { ganho de } r \text { peso } \\
\text { adequado } \\
\text { gestantes }\end{array}$ & $\begin{array}{l}\text { O ganho de peso adequado } \\
\text { foi apresentado em } 27,6 \% \\
\text { das gestantes que tiveram } \\
\text { intervenção e } 25,4 \% \text { das } \\
\text { gestantes que não tiveram } \\
\text { intervenção. } \\
\text { Não encontrando uma } \\
\text { diferença significativa. }\end{array}$ \\
\hline A6 & $\begin{array}{l}\text { Avaliando a divisão digital entre } \\
\text { mulheres perinatais de baixa } \\
\text { renda: } \\
\text { Oportunidades para } \\
\text { fornecimento de informações e } \\
\text { aconselhamento em saúde }\end{array}$ & $\begin{array}{l}\text { Acquavita SP. et } \\
\text { al. } \\
\text { EUA } \\
2018\end{array}$ & $\begin{array}{l}\text { Explorar interesses, } \\
\text { atitudes } \\
\text { preocupações da } \\
\text { população em relação } \\
\text { a tecnologia para } \\
\text { fornecer informações } \\
\text { e intervenções de } \\
\text { saúde }\end{array}$ & $\begin{array}{l}88 \% \text { dos participantes } \\
\text { relataram usar a Internet } \\
\text { para pesquisar informações } \\
\text { sobre gravidez, } \\
\text { amamentação, paternidade, } \\
\text { saúde infantil e nutrição. } \\
30 \% \text { relataram usar um } \\
\text { produto relacionado à } \\
\text { saúde ou de autoajuda. }\end{array}$ \\
\hline
\end{tabular}




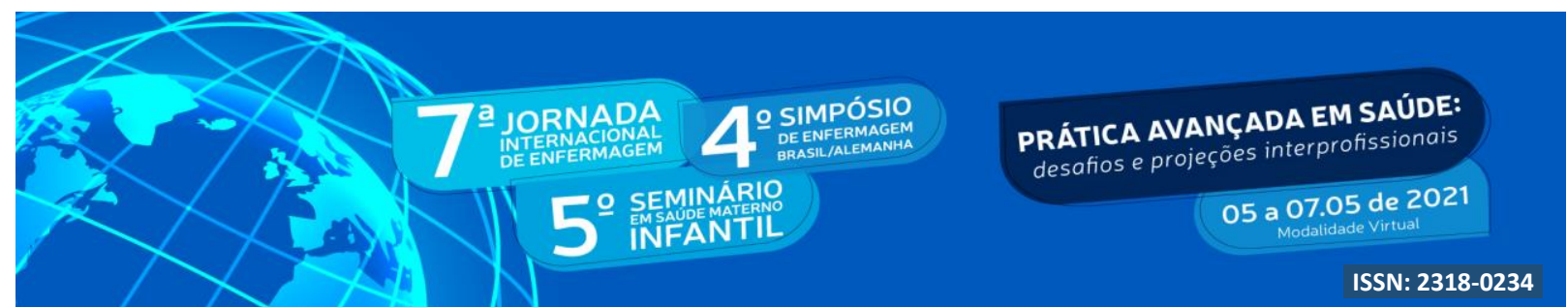

\begin{tabular}{|c|c|c|c|c|}
\hline A7 & $\begin{array}{l}\text { Impacto das visitas domiciliares } \\
\text { pré-natais assistidas por } \\
\text { smartphone no uso de partos por } \\
\text { mulheres }\end{array}$ & $\begin{array}{l}\text { Hacket K. et al. } \\
\text { EUA } \\
2018\end{array}$ & 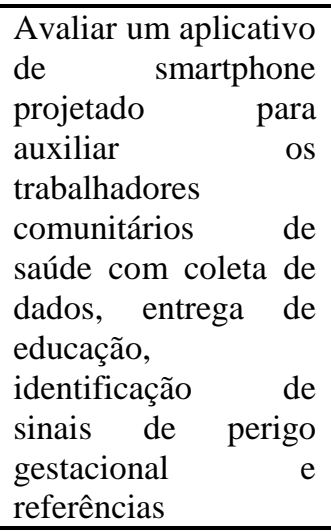 & $\begin{array}{l}\text { As chances de parto nas } \\
\text { instalações entre mulheres } \\
\text { aconselhadas por } \\
\text { profissionais de saúde por } \\
\text { meio de smartphones } \\
\text { tinham o dobro das } \\
\text { chances entre as mulheres } \\
\text { que viviam nas aldeias em } \\
\text { protocolos em papel. }\end{array}$ \\
\hline A8 & $\begin{array}{lrr}\text { Nutrição e exercícios } & \text { para } \\
\text { gravidez com suporte } & \text { de } \\
\text { aplicativo para smartphone } & \end{array}$ & $\begin{array}{l}\text { Kennelly MA. } \\
\text { et al. } \\
\text { EUA } \\
2018\end{array}$ & $\begin{array}{lrr}\text { Avaliar o } & \text { efeito } & \text { de } \\
\text { um estilo } & \text { de } & \text { vida } \\
\text { saudável } & \text { e } & \text { a } \\
\text { incidência } & & \text { de } \\
\text { mulheres } & & \text { com } \\
\text { diabetes } & \text { mellitus } \\
\text { gestacional } & \text { em } \\
\text { sobrepeso e obesas. }\end{array}$ & $\begin{array}{l}\text { A intervenção com suporte } \\
\text { de tecnologia móvel não } \\
\text { diminuiu a incidência de } \\
\text { diabetes } \\
\text { gestacional. Resultou em } \\
\text { benefícios } \\
\text { apoiando a dieta, exercício } \\
\text { físico e acompanhamento } \\
\text { no pré-natal. }\end{array}$ \\
\hline A9 & $\begin{array}{l}\text { O impacto de um sistema de } \\
\text { feedback diário baseado em } \\
\text { smartphone entre mulheres com } \\
\text { diabetes gestacional na } \\
\text { conformidade, controle } \\
\text { glicêmico, satisfação e resultado } \\
\text { da gravidez }\end{array}$ & $\begin{array}{l}\text { Miremberg MD. } \\
\text { et al. } \\
\text { EUA } \\
2018\end{array}$ & 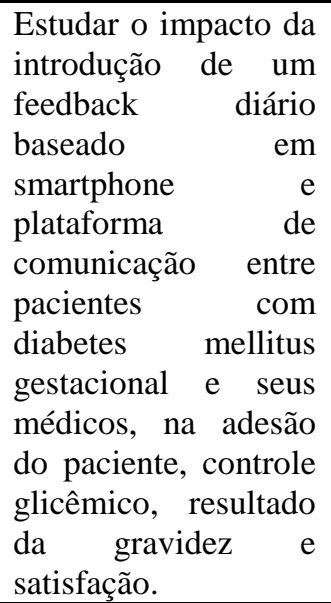 & $\begin{array}{l}\text { A glicose } \\
\text { significativamente menor } \\
\text { no grupo com intervenção } \\
\text { do smartphone em } \\
\text { comparação com o grupo } \\
\text { controle }(105,1 \pm 8,6 \mathrm{mg} / \\
\text { dL vs. 112,6 } \pm 7,4 \mathrm{mg} / \\
\mathrm{dL} \text { p }<0,001) \text {. A taxa geral } \\
\text { de insulina, o tratamento } \\
\text { foi menor no grupo de } \\
\text { smartphones em } \\
\text { comparação com o grupo } \\
\text { de controle (13,3\% vs. } \\
30,0 \%, p=0,044)\end{array}$ \\
\hline $\mathbf{A 1 0}$ & $\begin{array}{l}\text { Adaptação transcultural do } \\
\text { aplicativo Zero Mothers Die } \\
\text { para dispositivos móveis } \\
\text { no Brasil: contribuições para a } \\
\text { saúde digital com abordagem do } \\
\text { cuidado } \\
\text { centrado na e-gestante. }\end{array}$ & $\begin{array}{l}\text { Silva AB. et al. } \\
\text { Brasil } \\
2019\end{array}$ & \begin{tabular}{lr}
\multicolumn{2}{l}{ Descrever o processo } \\
de adaptação & do \\
aplicativo & para \\
combate & à \\
mortalidade & \\
materna & Zero \\
Mothers Die &
\end{tabular} & $\begin{array}{l}\text { Realizado alterações no } \\
\text { conteúdo do aplicativo. } \\
\text { Quanto à relação entre a } \\
\text { gestante e o aplicativo em } \\
\text { busca de informação sobre } \\
\text { sua saúde o resultado foi } \\
\text { em geral satisfatório e } \\
\text { acima da média. }\end{array}$ \\
\hline
\end{tabular}




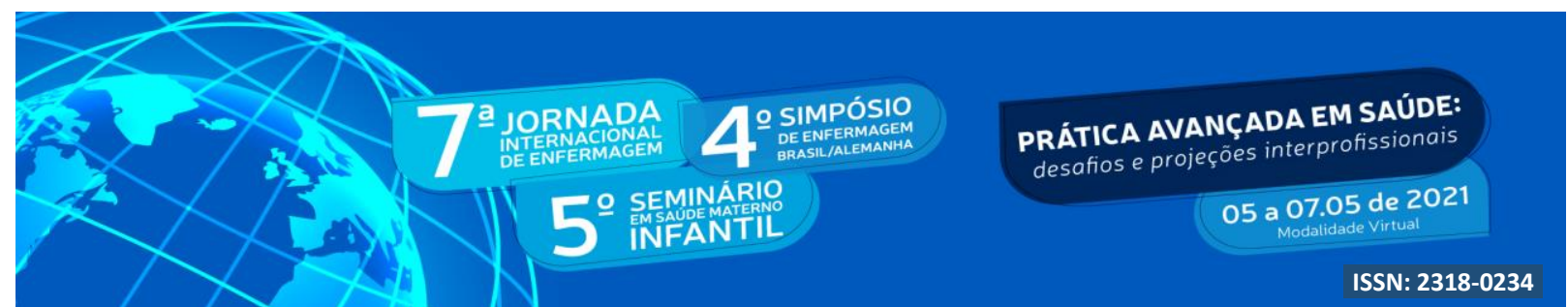

\begin{tabular}{|c|c|c|c|c|}
\hline A11 & $\begin{array}{l}\text { Compreendendo o } \text { uso de } \\
\text { aplicativos de smartphone para } \\
\text { informações de }\end{array}$ & $\begin{array}{l}\text { Wang N. et al. } \\
\text { Canadá } \\
2019\end{array}$ & $\begin{array}{l}\text { Investigar o uso de } \\
\text { aplicativos por } \\
\text { mulheres chinesas } \\
\text { durante a gravidez e } \\
\text { obter uma melhor } \\
\text { compreensão de seus } \\
\text { pontos de vista e } \\
\text { atitudes em relação a } \\
\text { aplicativos que } \\
\text { contêm informações } \\
\text { de saúde. }\end{array}$ & $\begin{array}{l}\text { O principal motivo para } \\
\text { usar um aplicativo era } \\
\text { monitorar } \\
\text { desenvolvimento do feto } \\
(436 / 535,81,5 \%) \text {, seguido } \\
\text { de aprendizagem sobre } \\
\text { nutrição e registro da dieta } \\
\text { na gravidez (140/535, } \\
26,2 \%) \text {. }\end{array}$ \\
\hline A12 & $\begin{array}{l}\text { Melhorando o conhecimento de } \\
\text { mulheres grávidas usando um } \\
\text { aplicativo de pré-eclâmpsia }\end{array}$ & $\begin{array}{l}\text { Parsa S. et al. } \\
\text { Holanda } \\
2019\end{array}$ & $\begin{array}{l}\text { Investigar o efeito de } \\
\text { um aplicativo móvel } \\
\text { de pré-eclâmpsia no } \\
\text { conhecimento das } \\
\text { gestantes }\end{array}$ & $\begin{array}{l}\text { Houve um aumento } \\
\text { significativo de } 63 \% \text { na } \\
\text { pontuação média de } \\
\text { conhecimento após a } \\
\text { intervenção. O uso de um } \\
\text { aplicativo educacional } \\
\text { melhora o conhecimento } \\
\text { de mulheres grávidas sobre } \\
\text { pré-eclâmpsia. }\end{array}$ \\
\hline A13 & $\begin{array}{l}\text { Um aplicativo móvel de cuidado } \\
\text { pré-natal para reduzir as visitas } \\
\text { pessoais }\end{array}$ & $\begin{array}{l}\text { Marko KI. et al. } \\
\text { Canadá } \\
2019\end{array}$ & $\begin{array}{l}\text { Testar a eficácia de } \\
\text { um aplicativo móvel } \\
\text { de assistência pré- } \\
\text { natal para facilitar } \\
\text { uma redução de } \\
\text { agendamento de } \\
\text { visitas presenciais } \\
\text { para gestações de } \\
\text { baixo risco. }\end{array}$ & $\begin{array}{l}\text { O grupo experimental teve } \\
\text { significativamente menos } \\
\text { cuidados pré-natais Visitas } \\
\text { ( } 7,9 \text { visitas) do que os } \\
\text { pacientes do grupo } \\
\text { controle ( } 10,2 \text { visitas). O } \\
\text { uso de um aplicativo } \\
\text { móvel de cuidado pré-natal } \\
\text { foi associado à redução de } \\
\text { visitas presenciais, e não } \\
\text { houve redução } \\
\text { na satisfação do paciente } \\
\text { ou do provedor. }\end{array}$ \\
\hline A14 & $\begin{array}{l}\text { Uso de tecnologia móvel para o } \\
\text { cuidado gestacional: avaliação } \\
\text { do aplicativo GestAção }\end{array}$ & $\begin{array}{l}\text { Silva RM. et al. } \\
\text { Brasil } \\
2019\end{array}$ & $\begin{array}{l}\text { Avaliar o aplicativo } \\
\text { GestAção, com base } \\
\text { na experiência de uso } \\
\text { das gestantes }\end{array}$ & $\begin{array}{l}\text { O estudo evidenciou } \\
\text { significativo nível de } \\
\text { satisfação das gestantes } \\
\text { com o uso do aplicativo, } \\
\text { considerando os objetivos } \\
\text { (IVC= }=0,92) \text {, estrutura e } \\
\text { apresentação (IVC=0,86), } \\
\text { e relevância (IVC=0,92). }\end{array}$ \\
\hline
\end{tabular}

Fonte: Elaborado pelos autores. Brasil, Rio Branco, 2020.

Os artigos estudados evidenciaram melhorias no cuidado e na vida das mulheres que utilizavam os aplicativos. Informando conteúdos sobre a gestação de forma educativa, o que pode melhorar e fortalecer o conhecimento durante esta fase. Todos os estudos mostraram os benefícios gerados com o uso da plataforma digital, em geral de forma satisfatória.

Os aplicativos mostraram alguns benefícios relacionados ao engajamento das mulheres nas consultas de rotina do pré-natal. Um estudo mostrou que as pacientes que faziam o uso do aplicativo para orientações, se mostraram 


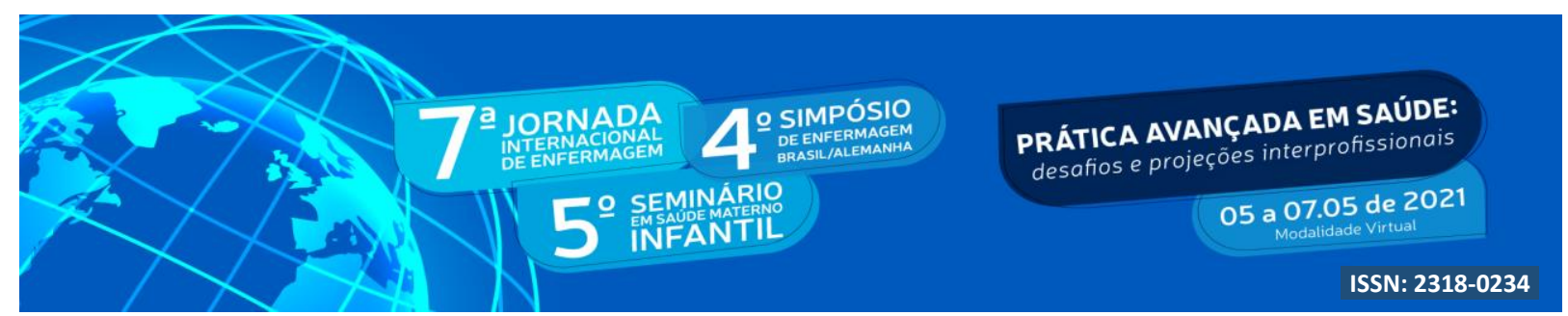

mais engajadas e menos faltosas as reuniões e consultas. (LEDFORD et al. 2015)

Algumas mulheres indicaram que o uso do aplicativo do smartphone auxiliou para que façam escolhas alimentares mais saudáveis e tenham tempo para praticar exercícios, porém o uso do aplicativo não foi associado a melhorias significativas na ingestão alimentar durante a maternidade. (DODD et al. 2018)

Um estudo mostrou a importância de um aplicativo para orientar mulheres tabagistas. Durante o estudo as mulheres relataram que o conteúdo ajudou a entender os riscos do tabaco. Inclusive que algumas orientações ajudaram a manifestar o interesse de parar de fumar. (DOTSON et al. 2017)

O uso dos aplicativos móveis se tornam apropriados quando comparados a outros métodos, como por exemplo, orientação verbal e cartilhas educativas. Além de ter um acesso prático e fácil, algumas mulheres relatam a praticidade, por estar sempre disponível no smartphone. (BORGEN et al. 2017)

O profissional de saúde sempre busca maneiras para encorajar o comportamento saudável de seus pacientes. Durante a gestação, a mulher vê uma oportunidade de mudar seus hábitos alimentares, e busca apoio para esse novo comportamento. Há estudos que mostram a melhoria nos hábitos e comportamentos, depois de uma intervenção com o aplicativo móvel, ajudando o melhor conhecimento das grávidas com pré-eclâmpsia (PARSA et al. 2019).

A tecnologia é uma ferramenta potente na contribuição para a atenção no pré-natal. Os aplicativos podem impactar diretamente no processo de empoderamento durante a gestação (SILVA et al. 2019). Se trata de um ótimo aliado para dar continuidade no acompanhamento dessas mulheres que necessitam estar seguras desde o início da gestação até após o parto.

\section{CONCLUSÃO}

O presente estudo mostrou que as tecnologias educacionais são um importante instrumento tanto para os profissionais de saúde quanto para as usuárias, e em como o uso de aplicativos móveis podem ajudar mulheres nas dificuldades e dúvidas recorrentes durante a fase gravídica.

A utilização das tecnologias em aplicativos móveis é uma ferramenta útil para trabalhar as orientações e disseminar diversas informações direcionadas a esse público. A 


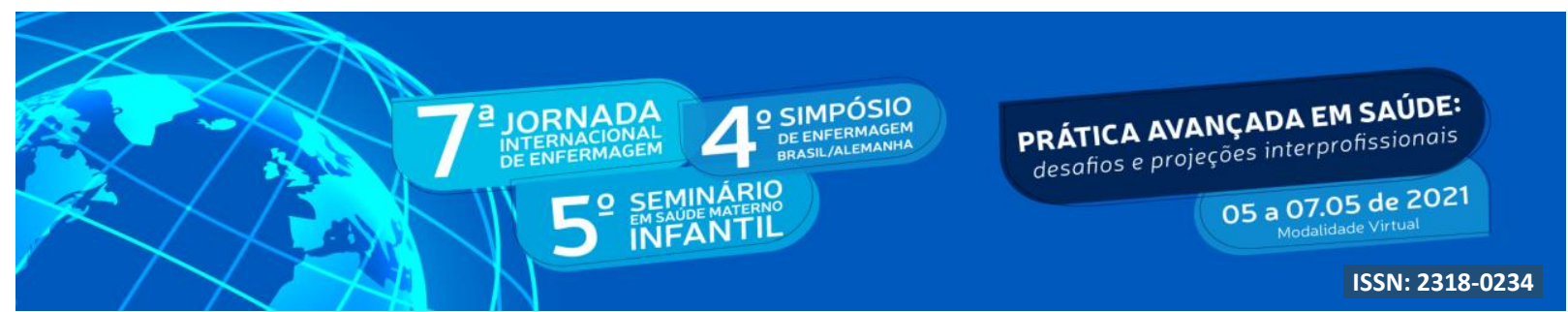

tecnologia nos smartphones é prática e possui uma facilidade de acesso. As orientações realizadas por meio das tecnologias complementaram os atendimentos presenciais, contribuíram para um melhor atendimento e favoreceram mudanças de hábitos prejudiciais à saúde.

Alguns estudos indicaram que lacunas precisam ser preenchidas quanto a qualidade de informações, eficácia das orientações, informações audiovisuais instrutivas e a usabilidade dos aplicativos móveis. Não foram encontrados estudos sobre tecnologia educacional relacionadas ao parto, onde muitas vezes são dúvidas frequentes durante a gestação. Dessa forma, emerge a necessidade de repensar sobre o desenvolvimento de aplicativos voltados para essa área.

\section{REFERENCIAS}

MENDONÇA, A. P. A. et al. A tecnologia atrelada ao resultado - Recursos Humanos frente as novas posturas e atribuições. Revista Razão Contábil e Finanças, v.8, n.2, p. 1-13, 2017.

KENNELLY, M. A. et al. Pregnancy, exercise and nutrition with smartphone application support: a randomized controlled trial. Obstetrics \& Gynecology, v. 131, n. 5, p. 818-826, 2018.

EDWARDS, E.A. et al. Gamification for health promotion: systematic review of behaviour change techniques in smartphones apps. BMJ Open, v. 6, n.10, p. 1-9, 2016.

CANDAU, V.M.F. Tecnologia Educacional: concepções e desafios. Cad Pesq [Internet]. 1979 28:616. [Acesso em: 18 abr. 2021]; Disponível em: http://www.fcc.org.br/pesquisa/publicacoes/c p/arquivos/386.pdf

MENDES, K.D.S, SILVEIRA, R.C.C.P, Galvão, C.M. Revisão integrativa: Método de pesquisa para a incorporação de evidências na saúde e na enfermagem. [Internet] Texto contexto - enferm. vol.17, n.4, pp.758-764. ISSN 1980-265X. 2008 [Acesso em: 7 dez. 2020]. Disponível em: http://dx.doi.org/10.1590/S0104-07072008000400018.

LEDFORD, C.J.W; CANZONA, M.R; CAFFERTY L.A; HODGE J.A. Mobile application as a prenatal education and engagement tool: A randomized controlled pilot. [Internet] Elsevier 2015; 578-582. Acesso em: 1 nov. 2020. Disponível em: http://dx.doi.org/10.1016/j.pec.2015.11.006.

BORGEN, I; GARNWEDNER-HOLME, L.M; JACOBSEN, A.F et al. Smartphone application for women with gestational diabetes mellitus: a study protocol for a multicentre randomised controlled trial. [Internet] BMJ Open 2017; 7: e013117. [Acesso em: 2 nov. 2020]. Disponível em: doi:10.1136/bmjopen-2016- 013117.

DODD, J.M; LOUISE J.; CRAMP, C. et al. Evaluation of a smartphone nutrition and physical activity application to provide lifestyle advice to pregnant women: The SNAPP randomised trial. [Internet] Matern Child Nutr. 2018;14:e12502.[Acesso em: 1 nov. 2020] Disponível em: $9^{\text {https://doi.org/10.1111/mcn.12502. }}$ 


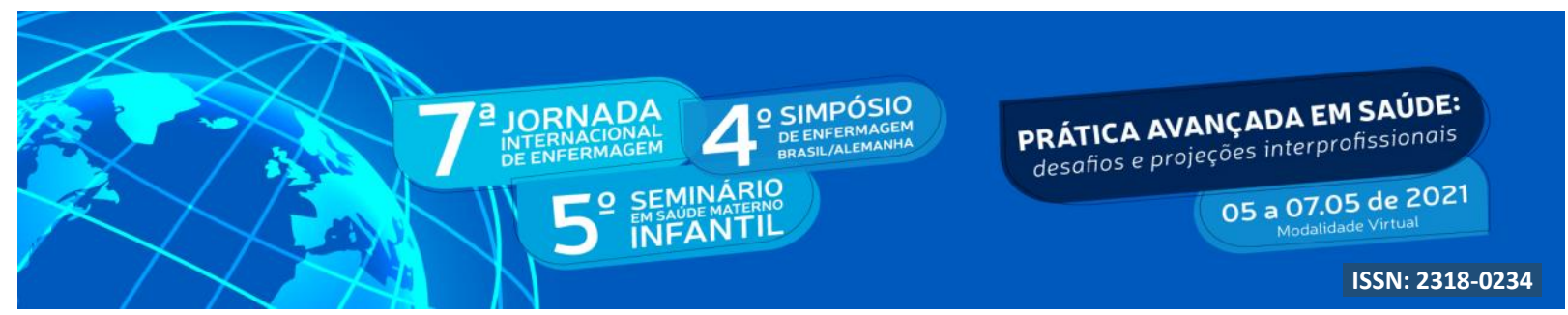

DOTSON, J.A.W; PINEDA, R.; CYLKOWSHKI, H; AMIRI, S. Development and Evaluation of an iPad Application to Promote Knowledge of Tobacco Use and Cessation by Pregnant Women. [Internet] Elsevier. 2017. V. 21. 174- 185. [Acesso em: 1 nov. 2020] Disponível em: https://doi.org/10.1016/j.nwh.2017.04.005.

TARQUI-MAMANI, C; SANABRIA-ROJAS, H; PORTUGAL-BENAVIDES, W.J. et al Eficacia de la tecnología móvil y ganancia de peso en gestantes en Callao, Perú. [Internet] Rev. Salud Pública. 20 (1): 67-72, 2018. [Acesso em: 2 nov. 2020]. Disponível em: https://doi.org/10.15446/rsap.V20n1.63488.

ACQUAVITA S.P; KRUMMEL, D.A; TALKS, A; COBB, A; MCCLURE, E. Assessing the Digital Divide Among Low-Income Perinatal Women: Opportunities for Provision of Health Information and Counseling. [Internet] Telemedicine and e-Health. Jan de 2019. 48-54. [Acesso em: 1 nov. 2020] Disponível em: https://doi.org/10.1089/tmj.2017.0292

HACKETT, K; LAFLEUR, C; NYELLA, P; GINSBURG, O; WENDY, L; SELLEN, D. Impact of smartphone- assisted prenatal home visits on women's use of facility delivery: Results from a clusterrandomized trial in rural Tanzania. [Internet] Plos One. 13(6): e0199400, 2018. Acesso em: 1 nov. 2020. Disponível em: https://doi.org/10.1371/journal.pone.0199400.

MIREMBERG, H; ARI-BEM, T; BETZER T. et al. The impact of a daily smartphone-based feedback system among women with gestational diabetes on compliance, glycemic control, satisfaction, and pregnancy outcome: a randomized controlled trial. [Internet] American Journal of Obstetrics and Gynecology. 2018. [Acesso em: 1 nov. 2020]. Disponível em: doi:10.1016/j.ajog.2018.01.044.

SILVA, A.B et al. Adaptação transcultural do aplicativo Zero Mothers Die para dispositivos móveis no Brasil: contribuições para a saúde digital com abordagem do cuidado centrado na $e$ gestante.[Internet]Rev. Bras. Saúde Mater. Infant., Recife, 19 (4): 763-775 out-dez., 2019. [Acesso em: 2 nov. 2020]. Disponível em: http://dx.doi.org/10.1590/1806-93042019000400002.

WANG, N; DENG, Z; WEN, L.M; DING, Y; Gengsheng H. Understanding the Use of Smartphone Apps for Health Information Among Pregnant Chinese Women: Mixed Methods Study.[Internet] JMIR Mhealth Uhealth. 2019. vol. 7.iss. 6. e12631. p. 14. [Acesso em: 1 nov. 2020]. Disponível em: http://mhealth.jmir.org/2019/6/e12631/.

PARSA, S; KHAJOUEI, R; BANESHI M.R; AALI, B.S. Improving the knowledge of pregnant women using a pre-eclampsia app: A controlled before and after study.[Internet] Elsevier. V 125 8690, 2019. [Acesso em: 2 nov. 2020] Disponível em: https://doi.org/10.1016/j.ijmedinf.2019.03.001.

MARKO, K; GANJU, N; KRAPF, J.M et al. A Mobile Prenatal Care App to Reduce In-Person Visits: Prospective Controlled Trial. [Internet] JMIR Mhealth Uhealth. 7(5):e10520. 2019. Acesso em: 2 nov. 2020. Disponível em: https://mhealth.jmir.org/2019/5/e10520/.

SILVA, R.M; BRASIL, C.C.P; BEZERRA I.C; QUEIROZ, F.F.S.N. Mobile health technology for gestational care: evaluation of the GestAcao's App. [Internet] Rev Bras Enferm. 2019;72(Suppl 3):266-73. Acesso em: 1 nov. 2020. Disponível em: http://dx.doi.org/10.1590/0034-7167-2018-0641. 